\section{Kompass \\ Onkologie}

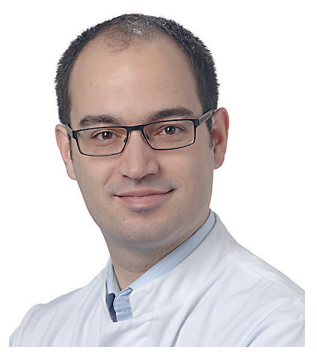

Klaus Metzeler

Labor für Leukämiediagnostik,

Medizinische Klinik und Poliklinik III, Klinikum der Universität München,

München, Deutschland

\title{
AML: Veränderte Therapielandschaft in optimale Versorgung umsetzen
}

Die Behandlung der akuten myeloischen Leukämie hat nach Jahren des gefühlten Stillstandes innerhalb kurzer Zeit einen tiefgreifenden Wandel durchlaufen. In den letzten 3 Jahren wurden in Europa mit den FLT3-Tyrosinkinaseinhibitoren Midostaurin und Gilteritinib, dem CD33-Antikörperkonjugat Gemtuzumab Ozogamicin und der liposomalen Chemotherapie-Formulierung CPX-351 vier neue Medikamente zur Therapie der AML zugelassen. In den USA sind es bereits 8 neue Substanzen. Dort haben neben den Inhibitoren der Isocitrat-Dehydrogenasen IDH1 und IDH2, Ivosidenib und Enasidenib, vor allem die Studien mit dem BCL2-Inhibitor Venetoclax binnen kürzester Zeit zu einer Veränderung der Therapielandschaft geführt [1]. Für Kombinationstherapien aus Venetoxclax mit hypomethylierenden Substanzen (HMA) oder niedrigdosiertem Cytarabin (LDAC) wurden in der Erstlinientherapie sehr hohe Ansprechraten berichtet $[2,3]$. Auch in Deutschland werden diese Kombinationstherapien deswegen an vielen Zentren, obwohl aktuell noch außerhalb der Zulassung, bereits regelhaft eingesetzt. Trotz der insgesamt, gerade im Vergleich zu einer intensiven Chemotherapie, besseren Verträglichkeit, gehen diese Behandlungsschemata aber durchaus mit spezifischen Toxizitäten und auch einem erheblichen Potential für Arzneimittelinteraktionen einher.
In dieser Ausgabe von «Kompass Onkologie» präsentieren wir Ihnen deshalb einen Übersichtsartikel, der sich mit dem Management von Venetoclax-Kombinationstherapien bei der AML im klinischen Alltag beschäftigt. Ein Fallbericht widmet sich zudem dem Einsatz von Venetoclax in der Zweitlinie bei HMA-refraktären Patienten. Während die Effektivität der BCL2-InhibitorTherapie bei der AML jenseits der Erstlinie insgesamt deutlich abnimmt, wirft dieser Artikel die Frage auf, ob wir mittels geeigneter Biomarker Patienten identifizieren können, die auch in der fortgeschrittenen Erkrankungssituation profitieren.

Angesichts der immer noch ungünstigen Prognose vor allem älterer AML Patienten ist die Entwicklung neuer, gut verträglicher und wirksamer therapeutischer Optionen natürlich hochwillkommen [4]. Zugleich erwachsen für uns Kliniker aus dieser Entwicklung aber auch neue Fragen und Herausforderungen.

Die Zulassung von Venetoclax in den USA umfasst beispielsweise Patienten im Alter ab 75 Jahren und solche, für die eine intensive Therapie nicht in Frage kommt. In manchen Kommentaren wird die Substanz bereits als neuer therapeutischer Standard für einen Großteil aller älteren AML Patienten dargestellt. Dabei sollten wir jedoch nicht vergessen, dass die FDA-Zulassung von Venetoclax (wie auch der IDH1/2-Inhibitoren) auf Basis einarmiger Studien, teils mit sehr begrenzten Patientenzahlen und mit kurzer Nachbeobachtungsdauer hinsichtlich des Überlebens, erfolgte $[2,3,5,6]$. Es ist daher noch unklar, ob die Venetoclax-basierten Kombinationen - anders als eine HMA- oder LDAC-Monotherapie - auch ein kuratives Potential aufweisen und welche Rolle in diesem Kontext die allogene Stammzelltransplantation bei Patienten mit gutem initialem Ansprechen spielt. Aktuellen Pressemitteilungen ist in diesem Zusammenhang zu entnehmen, dass in den noch nicht publizierten Phase-III-Studien (VIALE-A und VIALE-C) nur die Kombination aus Venetoclax und Azacitidin, nicht aber die Kombination mit LDAC, den primären Endpunkt eines verlängerten Gesamtüberlebens erreichen konnte.

Auf der anderen Seite sollten wir auch nicht vergessen, dass zumindest bei einer ausgewählten Subgruppe älterer AML Patienten (günstige Genetik, keine ausgeprägten Komorbiditäten) auch eine Induktions-Chemotherapie weiterhin eine Behandlungsalternative mit kurativem Potential darstellt [7]. Daher müssen für die verschiedenen neuen Substanzen trotz vielversprechender Ansprechraten zunächst die Überlebensdaten aus randomisierten Phase-IIIStudien und auch Subgruppenanalysen für die verschiedenen Risikogruppen und Genotypen abgewartet werden, bevor diese information@karger.com

() 2020 S.Karger GmbH, Freiburg

www.karger.com/kko

Karger ${ }^{\prime \prime}$ -
PD Dr. Klaus Metzeler

Labor für Leukämiediagnostik

Medizinische Klinik und Poliklinik III

Klinikum der Universität München, Campus Großhadern

Marchioninistrasse 15, 81377 München, Germany 
Therapien zum allgemein akzeptierten Standard werden.

Viele AML Patienten kommen aufgrund ihrer klinischen und genetischen Charakteristika für eine Behandlung mit mehreren der neu entwickelten Substanzen in Frage. Während noch vor wenigen Jahren die Therapieoptionen für ältere Patienten schon in der Erstlinie sehr begrenzt waren, stellt sich zukünftig deshalb die Frage nach der erfolgversprechendsten Therapiesequenz: Sollte ein älterer Patient mit sekundärer AML und Mutationen in FLT3 und IDH2 primär mit CPX-351, einer Venetoclax-basierten Therapie oder mit einer FLT3- oder IDH2-Inhibitor-enthaltenden Kombination therapiert werden? Was ist die beste weitere Behandlungsabfolge in der Zweit- und Drittlinie? Klinische Studien zur Beantwortung einiger dieser hochkomplexen Fragen werden von akademischen Studiengruppen auch in Deutschland vorangetrieben. Aufgrund der klinischen Heterogenität unseres Patientenkollektivs, der unterschiedli- chen Genotypen und der Vielzahl an möglichen Therapiesequenzen und Kombinationen werden viele Fragestellungen dennoch niemals in einer kontrollierten Studie untersucht werden. In dieser Situation kann die Analyse von Daten aus Patientenregistern einen wichtigen Beitrag zur Beantwortung relevanter klinischer Fragestellungen liefern. Um so wichtiger ist es daher, dass flächendeckend möglichst alle AML Patienten prospektiv in ein solches Register einer der multizentrischen deutschen Studiengruppen eingeschlossen werden auch wenn die Therapie zunehmend im ambulanten Sektor durchgeführt werden kann.

Ein weiterer, damit eng verknüpfter Aspekt ist das Biobanking. Die konsequente Archivierung von Biomaterialproben vom Zeitpunkt der Diagnosestellung, aber auch bei einem späteren Progress der Erkrankung, ist die unverzichtbare Grundlage für die Identifizierung neuer Biomarker, die uns zukünftig eine noch besser auf den einzelnen Pa- tienten zugeschnittene Therapie ermöglichen.

Deshalb gilt auch und gerade in Zeiten neuer, ambulant durchführbarer Therapien und vielfältiger Behandlungsoptionen, dass jeder AML Patient zumindest zum Zeitpunkt der Diagnosestellung und zu wichtigen therapeutischen Weichenstellungen in einem Schwerpunktzentrum mit Anbindung an eine AML-Studiengruppe vorgestellt werden sollte. Nur durch eine enge Zusammenarbeit und Vernetzung der niedergelassenen Hämatologen mit den akademischen Studiengruppen werden wir den wissenschaftlichen Fortschritt weiter unterstützen und in Zukunft eine optimale Versorgung für alle unsere AML Patienten gewährleisten können.

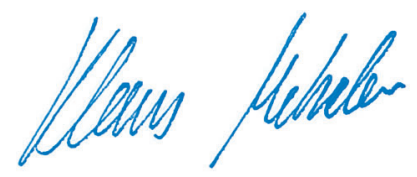

Klaus Metzeler

\section{Literatur}

1 DiNardo CD, Wei AH. How I treat acute myeloid leukemia in the era of new drugs. Blood. 2020;135(2):85-96.

-2 DiNardo CD, Pratz K, Pullarkat V, et al. Venetoclax combined with decitabine or azacitidine in treatment-naive, elderly patients with acute myeloid leukemia. Blood. 2019;133(1):7-17.

3 Wei AH, Strickland SA, Hou J-Z, et al. Venetoclax Combined With Low-Dose Cytarabine for Previously Untreated Patients With Acute Myeloid Leukemia: Results From a Phase Ib/II Study. J Clin Oncol. 2019;37(15):1277-1284.
4 Herold T, Rothenberg-Thurley M, Grunwald $\mathrm{VV}$, et al. Validation and refinement of the revised 2017 European LeukemiaNet genetic risk stratification of acute myeloid leukemia. Leukemia. 2020.

5 DiNardo CD, Stein EM, de Botton S, et al. Durable Remissions with Ivosidenib in IDH1-Mutated Relapsed or Refractory AML. N Engl J Med. 2018;378(25):2386-2398.
6 Stein EM, DiNardo CD, Pollyea DA, et al. Enasidenib in mutant IDH2 relapsed or refractory acute myeloid leukemia. Blood. 2017; 130(6):722-731.

7 Prassek VV, Rothenberg-Thurley M, Sauerland MC, et al. Genetics of acute myeloid leukemia in the elderly: mutation spectrum and clinical impact in intensively treated patients aged 75 years or older. Haematologica. 2018; 103(11):1853-1861. 